\title{
Staged bilateral pallidotomy for dystonic camptocormia: case report
}

\author{
Shiro Horisawa, MD, Mieko Oka, MD, Kotaro Kohara, MD, Takakazu Kawamata, MD, PhD, and \\ Takaomi Taira, MD, PhD
}

Department of Neurosurgery, Neurological Institute, Tokyo Women's Medical University, Shinjuku, Tokyo, Japan

\begin{abstract}
Camptocormia is a rare, involuntary movement disorder, presenting as truncal flexion while standing or walking, and is mainly observed as a feature of Parkinson's disease (PD) and primary dystonia. Deep brain stimulation (DBS) of the globus pallidus internus is effective for refractory camptocormia observed with PD or dystonia. However, the effectiveness of pallidotomy for camptocormia has not been investigated. The authors report the case of a 38-year-old man with anterior truncal bending that developed when he was 36 years old. Prior to the onset of the symptom, he had been taking antipsychotic drugs for schizophrenia. There were no features of PD; the symptom severely interfered with his walking and daily life. He was given anticholinergics, clonazepam, and botulinum toxin injections, which did not result in much success. Because of the patient's unwillingness to undergo implantation of a hardware device, he underwent staged bilateral pallidotomy with complete resolution for a diagnosis of tardive dystonic camptocormia. The Burke-FahnMarsden dystonia rating scale subscore for the trunk before and after bilateral pallidotomy was 3 and 0 , respectively. No perioperative adverse events were observed. Effects have persisted for 18 months. Bilateral pallidotomy can be a treatment option for medically refractory dystonic camptocormia without the need for device implantation.

https://thejns.org/doi/abs/10.3171/2018.5.JNS1840
\end{abstract}

KEYWORDS camptocormia; tardive dystonia; pallidotomy; functional neurosurgery

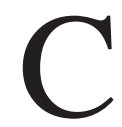

AMPTOCORMIA, also known as bent spine syndrome, is a rare, involuntary movement disorder presenting as truncal flexion while standing or walking, mainly observed as a feature of Parkinson's disease (PD) and primary dystonia. ${ }^{24}$ The pathophysiology of camptocormia associated with PD or dystonia is considered to be neurological in origin; treatment options include oral medications (levodopa, anticholinergics, and benzodiazepine) and botulinum toxin or lidocaine injections into affected muscles. ${ }^{18}$ However, effects of these treatments are usually limited and vary depending on the etiological diseases. For patients with dystonic camptocormia refractory to the these treatments, deep brain stimulation (DBS) of the globus pallidus internus (GPi) is effective. . $, 8,20,27^{2}$

Ablation of the GPi (pallidotomy) has an effect on dystonia that is similar to that of GPi-DBS on dystonia without the need for implantation of any device. ${ }^{6}$ However, there have been limited reports of the use of pallidotomy for dystonia. To the best of our knowledge, this is the first report of bilateral pallidotomy for tardive dystonic camptocormia with a successful outcome.

\section{Case Report}

History and Presentation

This 38-year-old man had camptocormia that began when he was 36 years old. The patient was a salesman and needed to walk long distances. Prior to the onset of camptocormia, he had begun a regimen of antipsychotic drugs (risperidone $6 \mathrm{mg}$ ) for schizophrenia at the age of 33 years. He developed anterior truncal bending while walking, which severely interfered with his work and daily activities. His antipsychotic drug was changed from risperidone to paliperidone. However, the truncal symptoms remained. There was no rigidity, tremor, bradykinesia, or other features of PD. The patient was administered anticholinergics, clonazepam, and botulinum toxin injections based on a diagnosis of tardive truncal dystonia, but these

ABBREVIATIONS AC-PC = anterior commissure-posterior commissure; BFMDRS = Burke-Fahn-Marsden dystonia rating scale; DBS = deep brain stimulation; ECT = electroconvulsive therapy; GPi = globus pallidus internus; PD = Parkinson's disease; STN = subthalamic nucleus.

SUBMITTED January 8, 2018. ACCEPTED May 2, 2018.

INCLUDE WHEN CITING Published online October 19, 2018; DOI: 10.3171/2018.5.JNS1840. 

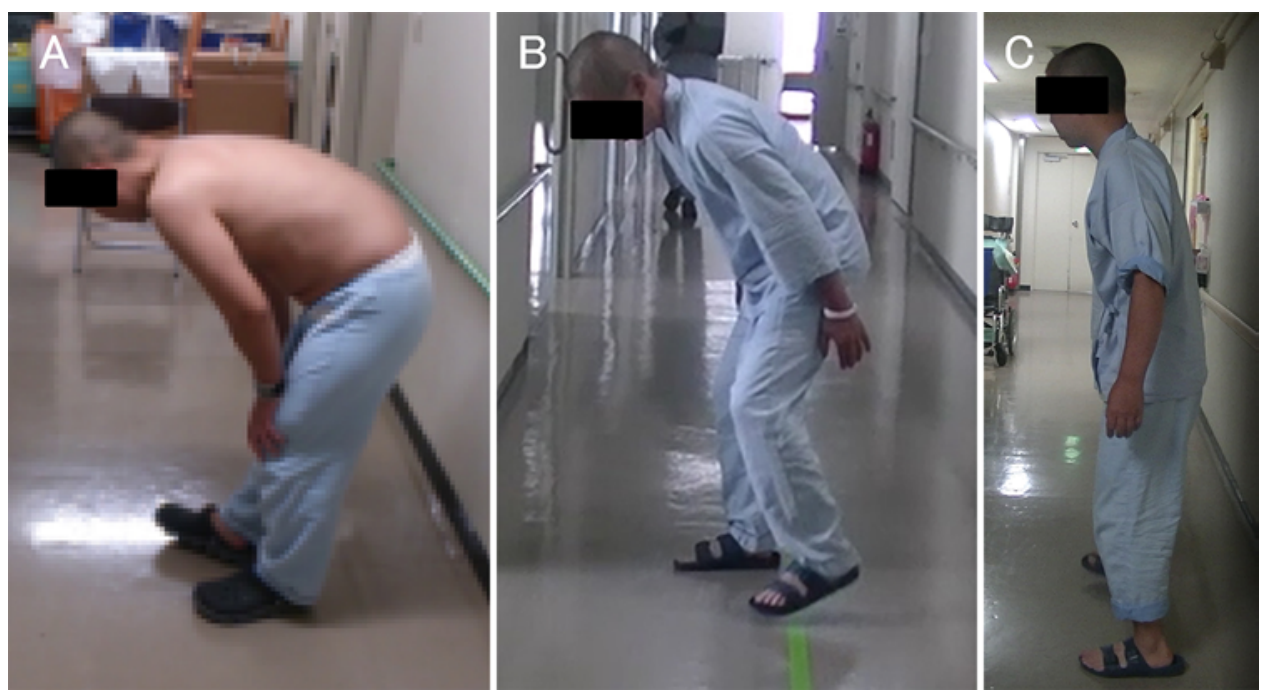

FIG. 1. Time course of the neurological condition. A: Preoperatively, anterior supine bending was severe. B: After left pallidotomy, camptocormia became mild but was still significant. C: Three days after bilateral pallidotomy, the camptocormia dramatically improved. Figure is available in color online only.

treatments were not effective. He was referred to our hospital for surgical treatment at which point he was administered paliperidone $(6 \mathrm{mg})$ and clotiazepam $(10 \mathrm{mg})$ for schizophrenia, and his psychiatric conditions were stable. Anterior truncal bending manifested only while walking (particularly when stepping forward using the right foot) but was not observed when running or in a supine position (Fig. 1A and Video 1).

VIDEO 1. Video clip showing the time course of symptomatic changes after left and right pallidotomy. Before left pallidotomy, the patient's camptocormia was severe and became mild, but was still significant, after left pallidotomy. With the addition of right pallidotomy performed 6 months after left pallidotomy, camptocormia dramatically improved. Copyright Shiro Horisawa. Published with permission. Click here to view.

The symptom was mild during the morning, and his family history was unremarkable. The patient's Burke-FahnMarsden dystonia rating scale (BFMDRS) score was 6 (movement scale [trunk] score 3 and disability scale [walk] score 3). Based on the onset after long-term antipsychotic use and the absence of any features of PD, we diagnosed tardive dystonic camptocormia. We suggested bilateral GPi-DBS to the patient, but he was not willing to undergo implantation of a hardware device and expressed deep concerns about possible ineligibility for electroconvulsive therapy (ECT) because of DBS. Therefore, we planned to perform staged bilateral pallidotomy.

\section{First Surgery and Postoperative Course}

Truncal bending manifested only when stepping forward using the right foot, which suggested that the right side was symptomatic dominant. Therefore, we first performed left-sided pallidotomy under local anesthesia. The stereotactic target in the left GPi was $20.0 \mathrm{~mm}$ lateral, 2.0 $\mathrm{mm}$ anterior, and $4.1 \mathrm{~mm}$ inferior to the midpoint of the anterior commissure-posterior commissure (AC-PC) line. The stereotactic target was adjusted based on MRI and CT imaging findings after the placement of a Leksell frame.
We used a monopolar radiofrequency probe $(1.0-\mathrm{mm}$ diameter tip with a 4.0-mm uninsulated length) and Leksell Neuro Generator (Elekta) for macrostimulation $(130 \mathrm{~Hz}$, $100-\mu s e c$ pulse width, up to $15 \mathrm{~mA}$ ) and coagulation. Microelectrode recordings were not used; rather, we verified the absence of capsular responses and visual phosphine with macrostimulation. Permanent lesions were created using thermocoagulation at $70^{\circ} \mathrm{C}$ for 40 seconds (Fig. 2). The electrode was then withdrawn in 2- and 4-mm increments to increase the lesion size. The day after surgery, the patient's anterior truncal bending significantly improved, with the camptocormia flexion angle changing from $70^{\circ}$ to $45^{\circ}$ (Fig. $1 \mathrm{~B}$ and Video 1 ). There were no perioperative adverse events. At 1 week postoperatively, the patient's BFMDRS score was 4 (movement scale [trunk] score 2; disability scale [walk] score 2). No adverse events were observed after the first surgery. Six months after the left pallidotomy, the symptom persisted and interfered with his walking. We then performed right-sided pallidotomy.

\section{Second Surgery and Postoperative Course}

The stereotactic target in the right GPi was $20.0 \mathrm{~mm}$ lateral, $2.0 \mathrm{~mm}$ anterior, and $4.1 \mathrm{~mm}$ inferior to the midpoint of the AC-PC line. Right-sided pallidotomy was performed in precisely the same manner as in the first surgery. No significant complications such as corticobulbar dysfunction were observed. Three days after right-sided pallidotomy, the symptom completely improved (BFMDRS score 0) without any deficit (Fig. 1C and Video 1). Head MRI performed 3 months after the right pallidotomy showed old lesions in the GPi bilaterally (Fig. 2). Eighteen months after the second surgery, the patient continued to be in a stable condition.

\section{Discussion}

This is the first report of dystonic camptocormia that was successfully treated with bilateral pallidotomy. Prior 


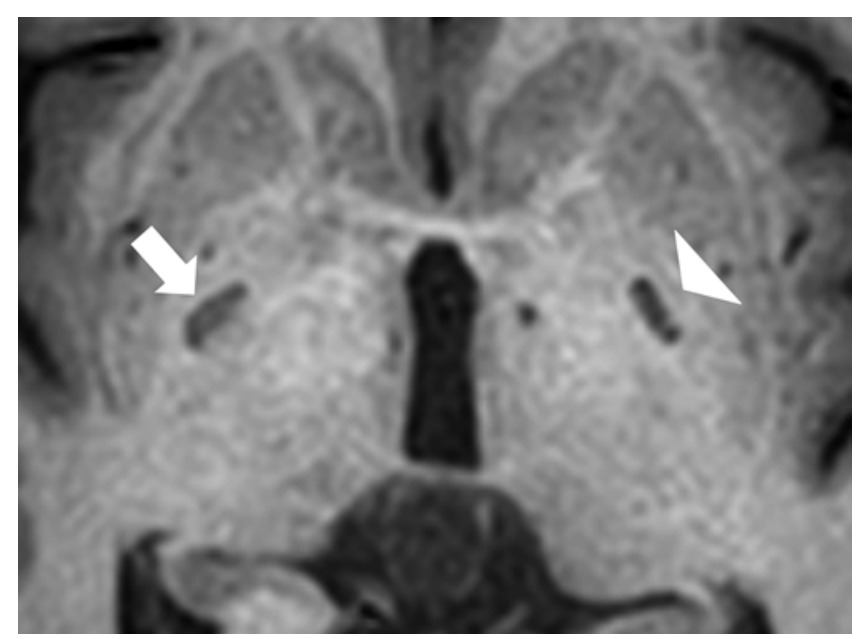

FIG. 2. Coronal MR image obtained 3 months after right pallidotomy, showing lesions in the left (arrowhead) and right (arrow) GPi.

treatment with antipsychotics and absence of PD symptoms suggested tardive dystonic camptocormia as a plausible diagnosis. Because of the patient's unwillingness to undergo device implantation, we selected bilateral pallidotomy as a treatment option, which ultimately resulted in complete resolution.

Camptocormia can have various kinds of causative backgrounds, including PD, dystonia, myopathy, dystrophy, and inflammation. ${ }^{18}$ Treatment of camptocormia usually depends on the etiological disease. Therefore, appropriate diagnosis is quite important. Camptocormia associated with PD or dystonia is a good candidate for DBS. Camptocormia has been most frequently reported as a feature of PD; subthalamic nucleus (STN)-DBS and GPi-DBS have been reported to be effective..$^{5,10,21,25}$ However, compared with camptocormia of dystonic origin, effects of DBS on camptocormia in PD tend to be heterogeneous. ${ }^{3-5,22,26}$ Capelle et al. reported on 7 patients (4 with dystonia and 3 with PD) treated with GPi-DBS or STNDBS. In the 4 patients with dystonia treated with GPiDBS, the mean improvement in the BFMDRS subscore for the trunk was $63 \%$ (range 50\%-67\%), whereas the 3 patients with PD, 2 treated with GPi-DBS and 1 treated with STN-DBS, showed 19.3\% improvement (range 0\%$33.3 \%){ }^{5}$ There have also been refractory cases unresponsive to DBS. 3,4,22,24,26 Prior atrophy or degeneration of the paraspinal vertebral muscle may be associated with the insufficient effect of DBS on camptocormia in PD. ${ }^{3}$

In dystonic camptocormia, there have been no reports of cases refractory to DBS. Summarizing reports with available BFMDRS data of symptomatic improvement, 13 patients received GPi-DBS and showed a mean improvement of $67.6 \%$ in BFMDRS score (range 33-100). 5,7 , $8,20,27$ Three patients experienced $100 \%$ improvement in their BFMDRS scores. ${ }^{7,20}$ The effects of DBS on dystonic camptocormia are expected to be better than those of DBS on PD camptocormia. Pallidotomy is expected to have effects on dystonic camptocormia that are similar to those of GPi-DBS on dystonic camptocormia, and the present study showed complete resolution following bilateral pallidotomy. Unilateral left-sided pallidotomy provided $33.3 \%$ improvement in the trunk BFMDRS score, and an additional right-sided pallidotomy 6 months after the first surgery resulted in complete resolution. The symptomatic improvements were confirmed within 1 week after the first and second surgery. In our experience, most patients with dystonia show symptomatic improvement within 1 week after the pallidotomy. ${ }^{11-13,16}$ On the contrary, in patients with GPi-DBS, it takes a few weeks or months for the dystonia to improve. We think that the difference in postoperative clinical course is attributed to surgical procedure, lesioning, or stimulation. However, the detailed mechanism of lesioning surgery in terms of faster clinical improvement remains unknown.

Tardive dystonia is a movement disorder that develops as a side effect of long-term administration of antipsychotic drugs. ${ }^{2}$ The onset site is predominantly the craniocervical region, whereas the trunk is a less common onset site. ${ }^{15}$ Tardive dystonia with truncal involvement mostly manifests as posterior truncal bending, and tardive dystonic camptocormia is rarely reported. ${ }^{19}$ Patients with tardive dystonia are good candidates for GPi-DBS and pallidotomy, whose effects may surpass those on primary dystonia., $916,17,23$ Among patients with tardive dystonia, use of antipsychotic drugs to treat schizophrenia or bipolar disorders is most common. ${ }^{15} \mathrm{ECT}$ is an important treatment option not only for schizophrenia but also for bipolar disorders or major depression. ${ }^{1}$ However, the safety of ECT in patients with an implanted DBS device has not been established. Pallidotomy can provide significant improvement in dystonia without the need for device implantation and, therefore, can be of significant value to treat patients with tardive dystonia while retaining their suitability for ECT.

Moreover, the present case was that of a relatively young patient (38 years old) and if this patient were to be treated using GPi-DBS, he would have required long-term management (over several decades) with battery replacement and adjustment of stimulation parameters, which would carry the risk of hardware-related complications, such as infection, battery malfunction, lead migration, or fracture. In a recent systematic review of hardware-related complications of DBS in 8983 patients, the overall risk of such complications was $11.75 \%$, which is not trivial. ${ }^{14}$ Implantation of mechanical devices can also result in psychological and physiological burden, particularly in young patients.

In conclusion, bilateral pallidotomy can be a treatment option for medically refractory dystonic camptocormia without the need for device implantation.

\section{References}

1. American Psychiatric Association: The Practice of Electroconvulsive Therapy: Recommendations for Treatment, Training, and Privileging. American Psychiatric Association, 2001

2. Aquino CC, Lang AE: Tardive dyskinesia syndromes: current concepts. Parkinsonism Relat Disord 20 (Suppl 1):S113-S117, 2014

3. Asahi T, Taguchi Y, Hayashi N, Hamada H, Dougu N, Takashima $\mathrm{S}$, et al: Bilateral subthalamic deep brain stimulation for camptocormia associated with Parkinson's disease. Stereotact Funct Neurosurg 89:173-177, 2011

4. Azher SN, Jankovic J: Camptocormia: pathogenesis, clas- 
sification, and response to therapy. Neurology 65:355-359, 2005

5. Capelle HH, Schrader C, Blahak C, Fogel W, Kinfe TM, Baezner H, et al: Deep brain stimulation for camptocormia in dystonia and Parkinson's disease. J Neurol 258:96-103, 2011

6. Cersosimo MG, Raina GB, Piedimonte F, Antico J, Graff P, Micheli FE: Pallidal surgery for the treatment of primary generalized dystonia: long-term follow-up. Clin Neurol Neurosurg 110:145-150, 2008

7. Fukaya C, Otaka T, Obuchi T, Kano T, Nagaoka T, Kobayashi K, et al: Pallidal high-frequency deep brain stimulation for camptocormia: an experience of three cases. Acta Neurochir Suppl 99:25-28, 2006

8. Hagenacker T, Gerwig M, Gasser T, Miller D, Kastrup O, Jokisch D, et al: Pallidal deep brain stimulation relieves camptocormia in primary dystonia. J Neurol 260:18331837,2013

9. Hashimoto T, Naito K, Kitazawa K, Imai S, Goto T: Pallidotomy for severe tardive jaw-opening dystonia. Stereotact Funct Neurosurg 88:105-108, 2010

10. Hellmann MA, Djaldetti R, Israel Z, Melamed E: Effect of deep brain subthalamic stimulation on camptocormia and postural abnormalities in idiopathic Parkinson's disease. Mov Disord 21:2008-2010, 2006

11. Horisawa S, Goto S, Takeda N, Takano Y, Kawamata T, Taira T: Pallidotomy for writer's cramp after failed thalamotomy. Stereotact Funct Neurosurg 94:129-133, 2016

12. Horisawa S, Goto S, Takeda N, Terashima H, Kawamata $\mathrm{T}$, Taira T: Bilateral pallidotomy for cervical dystonia after failed selective peripheral denervation. World Neurosurg 89:728.e1-728.e4, 2016

13. Horisawa S, Kawamata T, Taira T: Unilateral pallidotomy for blepharospasm refractory to botulinum toxin injections. Eur J Neurol 24:e39-e40, 2017

14. Jitkritsadakul O, Bhidayasiri R, Kalia SK, Hodaie M, Lozano AM, Fasano A: Systematic review of hardware-related complications of deep brain stimulation: do new indications pose an increased risk? Brain Stimul 10:967-976, 2017

15. Kiriakakis V, Bhatia KP, Quinn NP, Marsden CD: The natural history of tardive dystonia. A long-term follow-up study of 107 cases. Brain 121:2053-2066, 1998

16. Kohara K, Taira T, Horisawa S, Hanada T, Kawamata T: [Bilateral pallidotomy for tardive dystonia: a case report.] No Shinkei Geka 45:971-976, 2017 (Jpn)

17. Lenders MW, Buschman HP, Vergouwen MD, Steur EN, Kölling P, Hariz M: Long term results of unilateral posteroventral pallidotomy for antipsychotic drug induced tardive dyskinesia. J Neurol Neurosurg Psychiatry 76:1039, 2005

18. Margraf NG, Wrede A, Deuschl G, Schulz-Schaeffer WJ: Pathophysiological concepts and treatment of camptocormia. J Parkinsons Dis 6:485-501, 2016

19. Nandi D, Parkin S, Scott R, Winter JL, Joint C, Gregory R, et al: Camptocormia treated with bilateral pallidal stimulation. J Neurosurg 97:461-466, 2002
20. Reese R, Knudsen K, Falk D, Mehdorn HM, Deuschl G, Volkmann J: Motor outcome of dystonic camptocormia treated with pallidal neurostimulation. Parkinsonism Relat Disord 20:176-179, 2014

21. Sako W, Nishio M, Maruo T, Shimazu H, Matsuzaki K, Tamura T, et al: Subthalamic nucleus deep brain stimulation for camptocormia associated with Parkinson's disease. Mov Disord 24:1076-1079, 2009

22. Schäbitz WR, Glatz K, Schuhan C, Sommer C, Berger C, Schwaninger M, et al: Severe forward flexion of the trunk in Parkinson's disease: focal myopathy of the paraspinal muscles mimicking camptocormia. Mov Disord 18:408-414, 2003

23. Sobstyl M, Ząbek M: Deep brain stimulation for intractable tardive dystonia: literature overview. Neurol Neurochir Pol 50:114-122, 2016

24. Tatu L, Bogousslavsky J: Camptocormia: new signs in an old syndrome. Front Neurol Neurosci 42:87-95, 2018

25. Thani NB, Bala A, Kimber TE, Lind CR: High-frequency pallidal stimulation for camptocormia in Parkinson disease: case report. Neurosurgery 68:E1501-E1505, 2011

26. Umemura A, Oka Y, Ohkita K, Yamawaki T, Yamada K: Effect of subthalamic deep brain stimulation on postural abnormality in Parkinson disease. J Neurosurg 112:1283-1288, 2010

27. Yadav R, Ansari AZ, Surathi P, Srinivas D, Somanna S, Pal P: Bilateral pallidal deep brain stimulation in idiopathic dystonic camptocormia. Neurol India 63:911-914, 2015

\section{Disclosures}

Takaomi Taira received consulting fees from St. Jude Japan and a speaking fee from Daiichi-Sankyo.

\section{Author Contributions}

Conception and design: Taira, Horisawa, Kawamata. Acquisition of data: Taira, Horisawa, Oka, Kohara. Analysis and interpretation of data: Taira, Horisawa. Drafting the article: Horisawa. Critically revising the article: Horisawa. Reviewed submitted version of manuscript: Horisawa. Approved the final version of the manuscript on behalf of all authors: Taira. Study supervision: Taira.

\section{Supplemental Information \\ Videos \\ Video 1. https://vimeo.com/276856921.}

\section{Correspondence}

Takaomi Taira: Neurological Institute, Tokyo Women's Medical University, Tokyo, Japan. ttaira@twmu.ac.jp. 